\title{
Temperature jump and velocity slip in the moment method
}

\author{
Henning Struchtrup ${ }^{1, \star}$, Wolf Weiss ${ }^{2, \star \star}$ \\ 1 Dipartimento di Matematica, Università di Catania, Viale A. Doria 6, 95125 Catania, Italia \\ 2 Technical University Berlin, FB 6, Sekr. HF2, Straße des 17. Juni, 10623 Berlin, Germany \\ Received September 2, 1999
}

The moment method of the kinetic theory requires boundary conditions for the moments. It is not possible to derive these in an easy manner from the boundary conditions for the phase density. The conservation laws of mass, momentum and energy give only five relations between the moments and the properties of the wall. Additional boundary conditions may be determined from the minimax principle for the entropy production which was recently proposed by Struchtrup \& Weiss [1]. These ideas are outlined for the case of 13 and 14 moments and Maxwell's boundary conditions for the phase density which lead to temperature jumps and velocity slip at walls. In particular, one-dimensional stationary heat transfer between two walls at rest is considered. The temperature jumps at the walls are shown to depend on the values of all moments in front of the wall. The results obtained by the minimax principle are compared with results obtained for the same problem by a minimum principle for the global entropy production and by the so-called kinetic schemes [2].

\section{Introduction}

It is well known that the laws of Navier-Stokes and Fourier are not able to properly describe processes in gases with large Knudsen numbers. One successful attempt to describe these rarefied gases is Grad's moment method which has inspired the development of the growing field of extended thermodynamics [3]-[12]. In the theory of moments and in extended thermodynamics the state space is not spanned by the fields of density $\varrho$, velocity $v_{i}$ and temperature $T$ alone [13] but is enlarged by non-equilibrium quantities e.g. the pressure deviator $p_{\langle i j\rangle}$, the heat flux $q_{i}$ and other quantities, the so-called higher moments, which do not have an intuitive physical interpretation.

Moment methods and extended thermodynamics derive first order partial differential equations in spacetime for the variables that span the state space. Thus, one will need a set of initial and boundary conditions in order to formulate and solve a proper initial-boundary value problem. For a long time the formulation of boundary conditions for the higher moments - those without intuitive interpretation - has not been possible.

Recently Struchtrup \& Weiss proposed a method for the determination of boundary conditions for stationary processes [1]. In their method the boundary conditions for higher moments follow from the postulate that the maximum of the local entropy production becomes minimal in stationary processes. This method is purely phenomenological and no additional input is needed from kinetic theory. Therefore it may be also used in phenomenological theories of extended thermodynamics.

\footnotetext{
* present address: IMA, 400 Lind Hall, 207 Church St. SE, Minneapolis, MN 55455, USA; e-mail: struchtr@ima.umn.edu
}

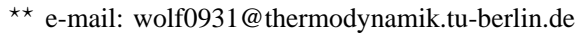


In the framework of the kinetic theory of gases, however, the formulation of boundary conditions does not present a problem in principle - Maxwell already proposed boundary conditions for the phase density and there are other models which describe the interaction between wall and gas more accurately $[14,15]$.

A temperature jump and a slip are observed at the wall, which depend on the details of the wallgas interaction and may be calculated within the kinetic theory [14, 15]. The phenomenological method of Struchtrup \& Weiss does not exhibit these features and the aim of the present paper is to connect the phenomenological method with the boundary conditions for the phase density. In particular we shall calculate the temperature jump and the slip velocity as functions of the moments.

In a previous paper the minimax principle was applied to a simple one dimensional heat conducting problem without temperature jumps at the walls [1]. Here we want to recalculate this problem with consideration of temperature jumps. Because we are dealing with higher moment theories, it is necessary to derive generalized equations for the temperature jump. Also generalized equations for the velocity slip are derived, which will be examined in future work.

Recently, LeTallec \& Perlat [16] have developed a method - called kinetic schemes - to solve the moment equations on the kinetic level. They solve the Boltzmann equation for a phase density that depends on space time only through the moments and maximizes the entropy [4,7]. This procedure allows the use of the boundary conditions for the phase density in their numerical calculations. This method was used in [2] for the calculation of stationary heat transfer with 13 and 14 moments and we shall compare our present results with those of [2].

The minimax principle of Struchtrup \& Weiss competes with the postulate that the global entropy production becomes minimal, due to Prigogine $[18,19]$. In the case of stationary heat conduction without temperature jumps, the latter gives unsatisfactory results for all Knudsen numbers [1]. This is not so when jumps are taken into account. In that case the results are unphysical for small Knudsen numbers but at large Knudsen numbers there are cases where the results from the global postulate are reasonable.

The paper is organized as follows: Sect. 2 gives a short survey on the moment method in kinetic theory and Maxwell's boundary conditions for the phase density. Moreover, the equations of extended thermodynamics with 13 and 14 fields are given. In Sect. 3 and 4 we derive the equations for temperature jumps and velocity slip for an arbitrary number of moments. These are specialized to the simple case of extended thermodynamics with 13 moments in Sect. 5. Section 6 deals with stationary heat transfer in the 14 moment case. Here, we use the afore mentioned principles for the entropy production in order to solve the differential equations. The paper ends with our conclusions. Some calculations of minor importance will be found in the appendix.

\section{Basic equations}

\subsection{Kinetic theory}

We consider mon-atomic ideal gases. The objective of the kinetic theory of gases is the determination of the phase density $f\left(x_{i}, t, c_{i}\right)$ which gives the number density of particles in the phase space element $d \mathbf{x} d \mathbf{c}$. Here, $x_{i}, t$ denote space and time variables, respectively, and $c_{i}$ is the velocity of a particle of mass $m$. The phase density is governed by the Boltzmann equation $[14,15]$,

$$
\frac{\partial f}{\partial t}+c_{k} \frac{\partial f}{\partial x_{k}}=\mathscr{S}(f)
$$

where the collision term $\mathscr{S}(f)$ accounts for the change of the phase density due to collisions among particles.

Once the phase density is known, one may calculate its moments, for instance the mass density $\varrho$, the momentum density $\varrho v_{i}$ and the energy density $\varrho \varepsilon$, given by

$$
\varrho=m \int f d \mathbf{c}, \varrho v_{i}=m \int c_{i} f d \mathbf{c}, \quad \varrho \varepsilon=\frac{3}{2} \varrho \frac{k}{m} T+\frac{\varrho}{2} v^{2}=\frac{m}{2} \int c^{2} f d \mathbf{c} .
$$

In these definitions, $k$ is Boltzmann's constant, $v_{i}$ denotes the barycentric velocity of the gas and $T$ denotes the temperature, which is thus defined by the mean kinetic energy of the particles. 


\subsection{Field equations of extended thermodynamics with 13 and 14 moments}

In moment methods one assumes that the state of the gas is satisfactorily described by a set of moments of the phase density

$$
u_{A}=\int \psi_{A}\left(c_{k}\right) f d \mathbf{c} .
$$

Extended thermodynamics of 14 variables chooses $\psi_{A}=m\left\{1, C_{i}, \frac{1}{2} C^{2}, C_{\langle i} C_{j\rangle}, \frac{1}{2} C^{2} C_{i}, C^{4}\right\}$ where $C_{i}=c_{i}-v_{i}$ is the peculiar velocity. This choice corresponds to the moments of the peculiar velocity ${ }^{1}$

$$
\begin{array}{ccc}
\varrho=m \int f d \mathbf{c}, & 0=m \int C_{i} f d \mathbf{c}, & \frac{3}{2} \varrho \frac{k}{m} T=\frac{m}{2} \int C^{2} f d \mathbf{c}, \\
p_{\langle i j\rangle}=m \int C_{\langle i} C_{j\rangle} f d \mathbf{c}, & q_{i}=\frac{m}{2} \int C^{2} C_{i} f d \mathbf{c}, & \Delta=m \int C^{4}\left(f-f_{M}\right) d \mathbf{c},
\end{array}
$$

where we have introduced the pressure tensor $p_{i j}$, the heat flux $q_{i}$ and the non-equilibrium part of the full trace of the fourth moment $\Delta . f_{M}$ denotes the local Maxwellian, viz.

$$
f_{M}=\frac{\varrho}{m} \sqrt{\frac{m}{2 \pi k T}}^{-3} e^{-\frac{m}{2 k T} C^{2}}
$$

and the trace of the pressure tensor defines the pressure

$$
p=\frac{1}{3} p_{i i}=\varrho \frac{k}{m} T .
$$

The equations for the moments follow by multiplication of the Boltzmann equation with $\psi_{A}$ and subsequent integration over velocity space. The resulting equations do not form a closed system of partial differential equations for the moments since they contain quantities which are not a priori related to the moments. Here, a closure assumption is required, and it is obvious that a phase density of the form

$$
f\left(x_{k}, t, c_{k}\right)=f\left(u_{A}\left(x_{k}, t\right), c_{k}\right)
$$

serves the purpose. A solution of this type is called "normal solution", and there are several methods to obtain it. Grad found his normal solution by an expansion around local equilibrium where the phase density is a Maxwellian. In the last years the method of maximizing the entropy [4] became more and more popular [7]. This method is equivalent to extended thermodynamics and we refer the interested reader to the monograph of Müller \& Ruggeri for a detailed discussion of these topics [5]

The normal solution corresponding to (3) follows either from Grad's method [3] or from entropy maximization $[4,5,7]$ and subsequent linearization around local thermal equilibrium as

$$
\begin{aligned}
f_{14}=f_{M}(1+ & \frac{m^{2}}{8 \varrho k^{2} T^{2}} \Delta-\frac{m^{3}}{12 \varrho k^{3} T^{3}} \Delta C^{2}+\frac{m^{4}}{120 \varrho k^{4} T^{4}} \Delta C^{4}- \\
& \left.+\frac{m^{2}}{2 \varrho k^{2} T^{2}} p_{\langle j k\rangle} C_{j} C_{k}-\frac{m^{2}}{\varrho k^{2} T^{2}} q_{k} C_{k}\left(1-\frac{1}{5} \frac{m}{k T} C^{2}\right)\right) .
\end{aligned}
$$

The moment equations read

$$
\begin{gathered}
\frac{\partial \varrho}{\partial t}+\frac{\partial \varrho v_{k}}{\partial x_{k}}=0 \\
\frac{\partial v_{i}}{\partial t}+v_{k} \frac{\partial v_{i}}{\partial x_{k}}+\frac{1}{\varrho} \frac{\partial p}{\partial x_{i}}+\frac{1}{\varrho} \frac{\partial p_{\langle i k\rangle}}{\partial x_{k}}=0
\end{gathered}
$$

\footnotetext{
${ }^{1}$ Angular brackets denote the traceless part of a symmetric tensor.
} 


$$
\begin{gathered}
\frac{3}{2} \varrho \frac{k}{m}\left(\frac{\partial T}{\partial t}+v_{k} \frac{\partial T}{\partial x_{k}}\right)+\frac{\partial q_{k}}{\partial x_{k}}+p_{k l} \frac{\partial v_{k}}{\partial x_{l}}=0 \\
\frac{\partial p_{\langle i j\rangle}}{\partial t}+\frac{\partial p_{\langle i j\rangle} v_{k}}{\partial x_{k}}+\frac{4}{5} \frac{\partial q_{\langle i}}{\left.\partial x_{j}\right\rangle}+2 p_{k\langle i} \frac{\partial v_{j\rangle}}{\partial x_{k}}=-\alpha \varrho p_{\langle i j\rangle}, \\
\frac{\partial q_{i}}{\partial t}+v_{k} \frac{\partial q_{i}}{\partial x_{k}}+\frac{5}{2} p \frac{k}{m} \frac{\partial T}{\partial x_{i}}+\frac{k T}{m} \frac{\partial p_{\langle i k\rangle}}{\partial x_{k}}+\frac{1}{6} \frac{\partial \Delta}{\partial x_{i}} \\
+\frac{7}{2} p_{\langle i k\rangle} \frac{k}{m} \frac{\partial T}{\partial x_{k}}-\frac{p}{\varrho} \frac{\partial i l\rangle}{\partial x_{k}}+\frac{7}{5} q_{i} \frac{\partial v_{k}}{\partial x_{k}}+\frac{7}{5} q_{k} \frac{\partial v_{i}}{\partial x_{k}}+\frac{2}{5} q_{k} \frac{\partial v_{k}}{\partial x_{i}}=-\frac{2}{3} \alpha \varrho q_{i}, \\
\frac{\partial \Delta}{\partial t}+v_{k} \frac{\partial \Delta}{\partial x_{k}}+8 \frac{k T}{m} \frac{\partial q_{k}}{\partial x_{k}}+8 \frac{k T}{m} p_{\langle k l\rangle} \frac{\partial v_{k}}{\partial x_{l}}-8 \frac{q_{k}}{\varrho} \frac{\partial p_{\langle k l\rangle}}{\partial x_{l}}=\frac{2}{7} \alpha \varrho \Delta \\
+28 \frac{\partial}{m} q_{k} \frac{\partial T}{\partial x_{k}}+\frac{\partial}{3} \Delta \frac{\partial v_{k}}{\partial x_{k}}=-\frac{1}{3} \alpha
\end{gathered}
$$

where $\alpha$ is a constant that follows by calculation of the collision production for Maxwell molecules. The first three equations are the equations of balance for the conserved quantities mass, momentum and energy.

The set of equations (7)-(12) reduces to Grad's 13 moment equations, if one sets $\Delta=0$ and omits the last equation.

\section{Boundary conditions}

For the calculation of boundary value problems with the systems given above, one needs boundary conditions for most of the moments. This is a problem - especially in the high moment theories - because in an experiment only few boundary values can be controlled. Furthermore, in a rarefied gas temperature jumps at the walls and a velocity slip can occur.

\subsection{Maxwell's boundary conditions}

The most simple model for the velocity and the temperature jump is due to Maxwell [14, 15]. He assumed that the fraction $\theta$ of the incident particles is thermalized at the wall and leaves the wall with a Maxwellian distribution. $\theta$ is called accommodation coefficient. The fraction $(1-\theta)$ of the emerging particles is reflected elastically at the wall.

We denote the velocity of a particle by $c_{i}$ and the velocity of the wall by $v_{i}^{W}$, such that the particle has the velocity $C_{i}^{W}=c_{i}-v_{i}^{W}$ in the frame where the wall is at rest. Moreover, we choose the normal vector $n_{i}$ of the wall so that it points into the gas. In this manner we have $n_{k} C_{k}^{W} \leq 0$ for the incident particles and $n_{k} C_{k}^{W} \geq 0$ for the particles that leave the wall.

Furthermore, $f_{N}\left(C_{i}^{W}, x_{i}, t\right)$ denotes the phase density in the gas close to the wall. For our purposes it is convenient to write $f_{N}$ as a function of the tangential velocity $C_{i}^{W}-n_{k} C_{k}^{W} n_{i}$ and the normal velocity $n_{k} C_{k}^{W}$ as $f_{N}\left(C_{i}^{W}-n_{k} C_{k}^{W} n_{i}, n_{k} C_{k}^{W}, x_{i}, t\right)$. In an elastic collision, the tangential velocity remains unchanged, while the normal velocity changes its sign. Such we have the phase densities $f_{N}\left(C_{i}^{W}-n_{k} C_{k}^{W} n_{i}, n_{k} C_{k}^{W}, x_{i}, t\right)$, for the incident particles $\left(n_{k} C_{k}^{W} \leq 0\right)$ and $f_{N}\left(C_{i}^{W}-n_{k} C_{k}^{W} n_{i},-n_{k} C_{k}^{W}, x_{i}, t\right)$ for the elastically reflected particles $\left(n_{k} C_{k}^{W} \geq 0\right)$, respectively. In the following, for simplicity of notation, we suppress the tangential velocity as well as space and time in the list of arguments of $f_{N}$ and write the phase density at the wall $\bar{f}$ according to Maxwells boundary conditions as

$$
\bar{f}= \begin{cases}\theta f_{W}+(1-\theta) f_{N}\left(-n_{k} C_{k}^{W}\right) & , \quad n_{k} C_{k}^{W} \geq 0 \\ f_{N}\left(n_{k} C_{k}^{W}\right) & , \quad n_{k} C_{k}^{W} \leq 0 .\end{cases}
$$


Here $f_{W}$ is the Maxwellian of the thermalized particles,

$$
f_{W}=f_{M}\left(\varrho_{W}, T_{W}, v_{i}^{W}\right)=\frac{\varrho_{W}}{m} \sqrt{\frac{m}{2 \pi k T_{W}}} e^{-\frac{m}{2 k T_{W}} C_{W}^{2}} .
$$

$T_{W}$ denotes the temperature of the wall and $\varrho_{W}$ is the density of the thermalized particles. $\varrho_{W}$ has to be determined so as to ensure that the wall does not accumulate particles, a condition which may be written in the form

$$
m \int_{n_{k} C_{k}^{W} \geq 0} C_{k}^{W} n_{k} \bar{f} d \mathbf{c}=-m \int_{n_{k} C_{k}^{W} \leq 0} C_{k}^{W} n_{k} \bar{f} d \mathbf{c} .
$$

We introduce the relative velocity, or slip velocity

$$
V_{k}=v_{k}-v_{k}^{W},
$$

which is parallel to the wall since there is no gas flow through the wall

$$
V_{k} n_{k}=0 .
$$

One aim of this paper is the determination of $V_{i}$.

In (15) we insert (13), substitute $-n_{k} C_{k}^{W} \rightarrow n_{k} C_{k}^{W}$ and introduce the peculiar velocity of the particles $C_{i}=C_{i}^{W}-V_{k}$ with $n_{k} C_{k}=n_{k} C_{k}^{W}$, because of (17). The mean value of $C_{i}$ vanishes by definition so that $\int C_{k} n_{k} f_{N} d \mathbf{c}=0$ and the condition (15) simplifies to

$$
m \int_{n_{k} C_{k}^{W} \geq 0} C_{k}^{W} n_{k} f_{W} d \mathbf{c}=m \int_{n_{i} C_{i} \geq 0} C_{k} n_{k} f_{N} d \mathbf{c} .
$$

\section{Temperature jump and slip}

In this section we derive equations for the temperature jump $T-T_{W}$ and the velocity slip $V_{i}$ at the wall. These follow from the jump conditions for the tangential force and the heat flux at the wall.

\subsection{Tangential force}

The tangential force $t_{i}=\left(p_{i j}-n_{i} p_{j k} n_{k}\right) n_{j}$ acting on the wall is determined by the phase density $\hat{f}$

$$
\hat{t}_{i}=m \int\left(c_{i}-n_{i} c_{k} n_{k}\right) c_{j} n_{j} \bar{f} d \mathbf{c}
$$

while the tangential force on the gas follows from the phase density $f_{N}$,

$$
t_{i}=m \int\left(c_{i}-n_{i} c_{k} n_{k}\right) c_{j} n_{j} f_{N} d \mathbf{c} .
$$

Both are equal due to Newton's third law and we evaluate this condition in the rest frame of the wall where $c_{i}=C_{i}^{W}$. With (13) we obtain

$$
\begin{aligned}
& m \int_{n_{k} C^{W} \geq 0}\left(C_{i}^{W}-n_{i} C_{k}^{W} n_{k}\right) C_{j}^{W} n_{j}\left[\theta f_{W}+(1-\theta) f_{N}\left(-n_{i} C_{i}^{W}\right)\right] d \mathbf{c}+ \\
& +m \int_{n_{k} C_{k}^{W} \leq 0}\left(C_{i}^{W}-n_{i} C_{k}^{W} n_{k}\right) C_{j}^{W} n_{j} f_{N} d \mathbf{c}=m \int\left(C_{i}^{W}-n_{i} C_{k}^{W} n_{k}\right) C_{j}^{W} n_{j} f_{N} d \mathbf{c} .
\end{aligned}
$$

Again, we substitute $-n_{k} C_{k}^{W} \rightarrow n_{k} C_{k}^{W}$ in the second term of the first integral and use $C_{k}^{W} n_{k}=C_{k} n_{k}$. We introduce the peculiar velocity $C_{i}=C_{i}^{W}-V_{i}$ in the integrals with $f_{N}$ and obtain with (18) and the definition of the pressure tensor (3) $)_{4}$ an equation for the determination of $V_{i}$, viz. 


$$
\begin{array}{r}
\frac{1-\theta}{\theta}\left(p_{i j}-n_{i} p_{j k} n_{k}\right) n_{j}=m \int_{n_{k} C_{k}^{W} \geq 0}\left(C_{i}^{W}-V_{i}-n_{i} C_{k}^{W} n_{k}\right) C_{j}^{W} n_{j} f_{W} d \mathbf{c} \\
-m \int_{n_{k} C_{k} \geq 0}\left(C_{i}-C_{k} n_{k} n_{i}\right) C_{j} n_{j} f_{N} d \mathbf{c} .
\end{array}
$$

Note that the tangential force vanishes in the case $\theta=0$, - there are no shear stresses at an elastically reflecting wall.

\subsection{Energy flux}

The normal part of the energy flux $\frac{m}{2} \int c^{2} c_{i} f d \mathbf{c}$ is continuous at the wall, a condition which we may write in the rest frame of the wall as

$$
\begin{aligned}
& \frac{m}{2} \int_{n_{k} C_{k}^{W} \geq 0} C_{W}^{2} C_{k}^{W} n_{k}\left[\theta f_{W}+(1-\theta) f_{N}\left(-n_{i} C_{i}\right)\right] d \mathbf{c}+ \\
& \frac{m}{2} \int_{n_{k} C_{k}^{W} \leq 0} C_{W}^{2} C_{k}^{W} n_{k} f_{N} d \mathbf{c}=\frac{m}{2} \int C_{W}^{2} C_{k}^{W} n_{k} f_{N} d \mathbf{c} .
\end{aligned}
$$

By means of the same substitutions as above this equation reduces to

$$
\begin{array}{r}
\frac{1-\theta}{\theta}\left(q_{k} n_{k}+V_{i} p_{\langle i k\rangle} n_{k}\right)=\frac{m}{2} \int_{n_{k} C_{k}^{W} \geq 0} C_{W}^{2} C_{k}^{W} n_{k} f_{W} d \mathbf{c}-V^{2} \theta \frac{m}{2} \int_{n_{k} C_{k}^{W} \geq 0} C_{k}^{W} n_{k} f_{W} d \mathbf{c} \\
-\frac{m}{2} \int_{n_{k} C_{k} \geq 0}\left(C^{2}+2 C_{i} V_{i}\right) C_{k} n_{k} f_{N} d \mathbf{c},
\end{array}
$$

where we have used (18) and the definitions of the pressure tensor and the heat flux (3) $)_{4,5}$. We eliminate $V^{2}$ by means of the scalar product of (19) with $V_{i}$ and obtain

$$
\begin{array}{r}
\frac{1-\theta}{\theta}\left(2 q_{k}+p_{\langle k r\rangle} V_{r}\right) n_{k}=m \int_{n_{k} C_{k}^{W} \geq 0} C_{W}^{2} C_{k}^{W} n_{k} f_{W} d \mathbf{c}-V_{i} m \int_{n_{k} C_{k}^{W} \geq 0} C_{i}^{W} C_{k}^{W} n_{k} f_{W} d \mathbf{c} \\
-m \int_{n_{i} C_{i} \geq 0} C^{2} C_{k} n_{k} f_{N} d \mathbf{c}-V_{i} m \int_{n_{i} C_{i} \geq 0} C_{i} C_{k} n_{k} f_{N} d \mathbf{c} .
\end{array}
$$

This equation will be used for the determination of the temperature jump. Note that an elastically reflecting wall $-\theta=0$ - is adiabatic, i.e. $q_{k} n_{k}=0$.

\subsection{Equations for temperature jump and slip}

It is an easy task to calculate the integrals over the Maxwellian distribution in $(18,19,20)$, see Appendix A. For further simplification we decompose the pressure tensor into its irreducible parts $p \delta_{i j}$ and $p_{\langle i j\rangle}$ and define half-space moments of $f_{N}$ by

$$
H_{i_{1} \cdots i_{n}}^{r}=m \int_{n_{i} C_{i} \geq 0} C^{2 r} C_{i_{1}} \cdots C_{i_{n}} f_{N} d \mathbf{c} .
$$

Thus, we may write the condition for conservation of mass (18) as

$$
\frac{1}{2} \sqrt{\frac{2}{\pi}} \varrho_{W} \sqrt{\frac{k T_{W}}{m}}=H_{k}^{0} n_{k} .
$$

This equation serves for the elimination of the density $\varrho_{W}$ in the conditions for the shear stress and the heat flux $(19,20)$ which now read

$$
\begin{aligned}
\frac{1-\theta}{\theta}\left(p_{\langle i k\rangle}-n_{i} p_{\langle j k\rangle} n_{j}\right) n_{k} & =-V_{i} H_{k}^{0} n_{k}-\left(\delta_{i j}-n_{i} n_{j}\right) n_{k} H_{j k}^{0}, \\
\frac{1-\theta}{\theta}\left(2 q_{k}+p_{\langle k r\rangle} V_{r}\right) n_{k} & =4 \frac{k T_{W}}{m} H_{k}^{0} n_{k}-H_{k}^{1} n_{k}-V_{i} H_{i k}^{0} n_{k} .
\end{aligned}
$$

We shall proceed with the evaluation of the equations (23). 


\section{Grad's 13 moment phase density}

The aim of this section is the determination of temperature jump and slip velocity in case that Grad's 13 moment density is appropriate for the description of the gas. This is the case in slowly varying processes with flat gradients.

\subsection{Phase density and boundary conditions}

Grad considered 13 moments to be relevant, the density $\varrho$, the velocity $v_{i}$, the temperature $T$, the pressure deviator $p_{\langle i j\rangle}$ and the heat flux $q_{i}$. By means of an expansion in Hermite polynomials he obtained the phase density [3][5]

$$
f_{13}=f_{M}\left(1+\frac{m^{2}}{2 \varrho k^{2} T^{2}} p_{\langle j k\rangle} C_{j} C_{k}-\frac{m^{2}}{\varrho k^{2} T^{2}} q_{k} C_{k}\left(1-\frac{1}{5} \frac{m}{k T} C^{2}\right)\right),
$$

where $f_{M}$ is the local Maxwellian (4). The same function $f_{13}$ follows from (6) by setting $\Delta=0$.

With (24) the conditions (23) for shear stress and heat flux read

$$
\begin{aligned}
\frac{2-\theta}{\theta}\left(p_{\langle i k\rangle}-n_{i} p_{\langle j k\rangle} n_{j}\right) n_{k} & =-\sqrt{\frac{2}{\pi}} \sqrt{\frac{m}{k T}}\left[V_{i}\left(\varrho \frac{k T}{m}+\frac{1}{2} p_{\langle i k\rangle} n_{i} n_{k}\right)+\frac{1}{5}\left(q_{i}-n_{i} q_{j} n_{j}\right)\right], \\
\frac{2-\theta}{\theta}\left(q_{k}+\frac{1}{2} p_{\langle k r\rangle} V_{r}\right) n_{k} & =\sqrt{\frac{2}{\pi}} \sqrt{\frac{m}{k T}}\left[2 \varrho \frac{k^{2}}{m^{2}} T\left(T_{W}-T\right)+\frac{k}{m}\left(T_{W}-\frac{3}{2} T\right) p_{\langle i k\rangle} n_{i} n_{k}-\frac{1}{10} V_{j} q_{j}\right] .
\end{aligned}
$$

The calculation of the half-space moments of $f_{13}$ is outlined in Appendix B.

\subsection{Temperature jumps}

We consider one-dimensional stationary heat transfer in a gas at rest between two walls of distance $L$, such that $v_{i}=v_{i}^{W}=0$ and $p_{\langle i j\rangle}=0$. In this case $(25)_{1}$ is identically fulfilled and $(25)_{2}$ reduces to

$$
T_{W}-T=\frac{2-\theta}{\theta} \sqrt{\frac{\pi}{2}} \frac{1}{2 \varrho \frac{k}{m}} \sqrt{\frac{m}{k T}} q_{k} n_{k},
$$

an equation that relates the temperature jump at the wall to the heat flux. Due to the energy balance (9) the heat flux is constant in this case,

$$
q=q_{k} n_{k}=\text { const }
$$

and the field equation for the heat flux (11) reduces to Fourier's law for Maxwell molecules, viz.

$$
\frac{15}{4} \frac{k^{2}}{m^{2}} T \frac{d T}{d x}=-\alpha q \text {. }
$$

We denote the temperatures of the gas - not the temperature of the wall! - at the left and the right wall by $T(x=0)=T_{0}$ and $T(x=L)=T_{L}$, respectively. With these boundary conditions we obtain from (27) the temperature function between the two walls as

$$
T=\sqrt{T_{0}^{2}+\left(T_{L}^{2}-T_{0}^{2}\right) \frac{x}{L}}
$$

accordingly the heat flux is given by

$$
q=-\frac{15}{8} \frac{k^{2}}{m^{2}} \frac{T_{L}^{2}-T_{0}^{2}}{\alpha L} .
$$


This would be the solution of the stationary heat flux problem, if the temperatures $T_{0}$ and $T_{L}$ were prescribed. But $T_{0}$ and $T_{L}$ are the temperatures of the gas at the walls and we are not able to prescribe these temperatures. Rather we may control the temperature of the walls, $T_{W_{0}}$ and $T_{W_{L}}$. Therefore we need a relation between $T_{0}$ and $T_{W_{0}}$ or between $T_{L}$ and $T_{W_{L}}$. These follow from equation (26) which relates the temperature difference between wall and gas to the heat flux as (note, that the normal vectors on the two walls point in opposite directions)

$$
T_{W_{0}}-T_{0}=\frac{2-\theta}{2 \theta} \sqrt{\frac{\pi}{2}} \frac{1}{\varrho_{0} \frac{k}{m}} \sqrt{\frac{m}{k T_{0}}} q_{k} n_{k} \quad, \quad T_{W_{L}}-T_{L}=-\frac{2-\theta}{2 \theta} \sqrt{\frac{\pi}{2}} \frac{1}{\varrho_{L} \frac{k}{m}} \sqrt{\frac{m}{k T_{L}}} q_{k} n_{k} .
$$

The pressure $p$ is constant in the process under consideration, see Eqn. (8). In (30) we introduce the heat flux (29) and dimensionless temperatures by $\hat{T}=T / T_{W_{0}}$ to obtain

$$
\frac{1-\hat{T}_{0}}{\hat{T}_{0}}=-\frac{2-\theta}{\theta} \sqrt{\frac{\pi}{2}} \frac{15}{16} K n \frac{\hat{T}_{L}^{2}-\hat{T}_{0}^{2}}{\sqrt{\hat{T}_{0}}} \quad, \quad \frac{\hat{T}_{W_{L}}-\hat{T}_{L}}{\hat{T}_{L}}=\frac{2-\theta}{\theta} \sqrt{\frac{\pi}{2}} \frac{15}{16} K n \frac{\hat{T}_{L}^{2}-\hat{T}_{0}^{2}}{\sqrt{\hat{T}_{L}}} .
$$

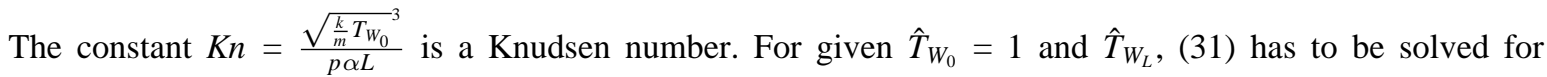
$\hat{T}_{0}, \hat{T}_{L}$ numerically in order to obtain the temperature (28) and the heat flux (29) which we may write in dimensionless form as

$$
\hat{T}=\sqrt{\hat{T}_{0}^{2}+\left(\hat{T}_{L}^{2}-\hat{T}_{0}^{2}\right) \hat{x}}, \hat{q}=\frac{q}{p \sqrt{\frac{k}{m} T_{W_{0}}}}=-\frac{15}{8} K n\left(\hat{T}_{L}^{2}-\hat{T}_{0}^{2}\right) .
$$

The temperature jumps (31) depend on the values of the accommodation coefficient $\theta$ and the Knudsen number $K n$ : there are large jumps for

i.) small values of the accommodation coefficient $\theta$, i.e. reflecting walls

ii.) large Knudsen numbers $K n$, i.e. mean free paths which are comparable to the distance $L$ of the walls.

This can be seen in Fig. 1, where the temperature is drawn for different values of the accommodation coefficient in a dense gas $(K n=0.001)$ and a rarefied gas $(K n=0.1)$. The wall temperatures are $\hat{T}_{W_{0}}=1$, and $\hat{T}_{W_{L}}=2$.
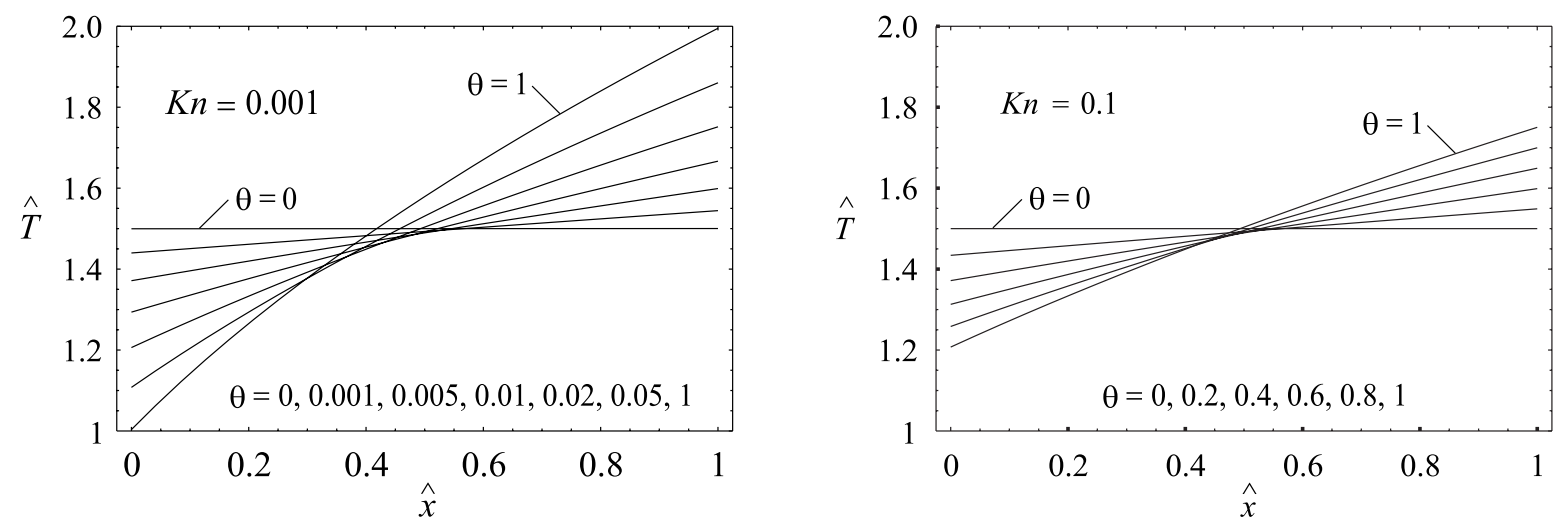

Fig. 1. Temperature $\hat{T}$ versus space coordinate $\hat{x}$.

Chapman \& Cowling [14] find for the temperature jump

$$
T_{W}-T=\frac{2-\theta}{\theta} u^{\prime} l \frac{\partial T}{\partial x}
$$

where $l=\frac{1}{\varrho \alpha} \sqrt{\frac{16 k T}{\pi m}}$ denotes the mean free path and $u^{\prime}$ is a number of order unity. We may identify $u^{\prime}$ by combining (26) and (27),

$$
u^{\prime}=\frac{15 \pi}{32 \sqrt{2}}=1.0413
$$


5.3 Slip

In this section we derive a formula for the velocity slip. We consider an isothermal shear flow with $T=T_{W}$, $p_{\langle j k\rangle} n_{j} n_{k}=0$ under the assumption that the heat flux is perpendicular to the flow, $q_{i}=q_{j} n_{j} n_{i}$. Now, the jump conditions (25) reduce to

$$
\begin{aligned}
V_{i} & =-\frac{2-\theta}{\theta} \sqrt{\frac{\pi}{2}} \sqrt{\frac{k T}{m}} \frac{p_{\langle i k\rangle} n_{k}}{p} \\
q_{k} n_{k} & =-\frac{1}{2} p_{\langle k r\rangle} V_{r} n_{k}=\frac{1}{2} \frac{\theta}{2-\theta} \sqrt{\frac{2}{\pi}} \varrho \sqrt{\frac{k T}{m}} V^{2} .
\end{aligned}
$$

It follows that the slip velocity $V_{i}$ is proportional to the shear stress at the wall. Eqn. (33) $)_{1}$ corresponds to (26) for the temperature jump. If we insert the Navier-Stokes law $p_{\langle i j\rangle}=-\frac{2 p}{\varrho \alpha} \frac{\partial v_{\langle i}}{\partial x_{k\rangle}}$, we find

$$
V_{i}=\frac{2-\theta}{\theta} u l \frac{\partial v_{\langle i}}{\partial x_{k\rangle}} n_{k}
$$

with $u=\frac{\pi}{2 \sqrt{2}}=1.107$ and one finds the same equation in the book by Chapman \& Cowling [14].

From $(33)_{2}$ we conclude that the process is isothermal only, if the power of the shear stress is removed by a heat flux. In this paper we do not consider a shear flow problem in detail; the equations for the slip velocity are derived for later use.

\section{Stationary heat transfer in extended thermodynamics with 14 fields}

In this section we consider extended thermodynamics with 14 fields where the state space is enlarged by the non-equilibrium part $\Delta$ of the full trace of the fourth moment. Now, the phase density is given by (6).

In the remainder of the paper, we restrict the argument to the case of stationary heat transfer in a gas at rest. Thus we have vanishing velocity and no slip. Similar problems with non-vanishing velocity, e.g. Couette flow, are more cumbersome, see [17], and may be considered in future work.

\subsection{Temperature jump}

In one-dimensional stationary heat transfer in a gas at rest we have from (7)-(12) $p_{\langle j k\rangle}=0, V_{i}=0$ and the jump condition (23) $)_{1}$ is identically fulfilled while the jump condition for the temperature $(23)_{2}$ reduces to

$$
\frac{1-\theta}{\theta} 2 q_{k} n_{k}=4 \frac{k T_{W}}{m} H_{k}^{0} n_{k}-H_{k}^{1} n_{k} .
$$

$H_{k}^{0}, H_{k}^{1}$ are half space moments of $f_{14}$ which are calculated in Appendix B as

$$
\begin{aligned}
H_{i}^{0} & =\frac{\varrho}{2} \sqrt{\frac{2}{\pi}} \sqrt{\frac{k T}{m}}\left(1-\frac{1}{120} \frac{\Delta}{p \frac{k T}{m}}\right) n_{i}, \\
H_{i}^{1} & =2 \varrho \sqrt{\frac{2}{\pi}} \sqrt{\frac{k T}{m}}\left(1+\frac{1}{40} \frac{\Delta}{p \frac{k T}{m}}\right) n_{i}+q_{i},
\end{aligned}
$$

so that (34) reduces to

$$
\frac{T_{W}-T}{T}=\frac{\frac{2-\theta}{2 \theta} \frac{1}{p} \sqrt{\frac{\pi}{2}} \sqrt{\frac{m}{k T}} q_{k} n_{k}+\frac{1}{30} \frac{\Delta}{p \frac{k T}{m}}}{1-\frac{1}{120} \frac{\Delta}{p \frac{k T}{m}}} .
$$

The temperature jump $T_{W}-T$ depends on all moments (temperature $T$, pressure $p$, heat flux $q_{k}$ and fourth moment $\Delta$ ) in front of the wall in the 14 moment case. 


\subsection{Field equations}

We consider the field equations (7)-(12) for one-dimensional stationary heat transfer. Here, all time derivatives and the velocity vanish. Thus, the mass balance (7) is identically fulfilled and the energy balance (9) reduces to $\frac{\partial q}{\partial x}=0$. Equation (10) now reads $\frac{8}{15} \frac{\partial q}{\partial x}=-\alpha \varrho p_{\langle 11\rangle}$ and accordingly $\left.p_{\langle i j\rangle}\right\rangle$ vanishes. The balance of momentum (8) gives the constancy of the pressure, $p=$ const , and this allows us to introduce the same dimensionless quantities as above, viz.

$$
\begin{aligned}
& \hat{x}=\frac{x}{L}, \quad \hat{T}=\frac{T}{T_{W_{0}}}, \quad \hat{\Delta}=\frac{\Delta}{p \frac{k}{m} T_{W_{0}}}, \\
& \hat{\varrho}=\frac{\varrho \frac{k}{m} T_{W_{0}}}{p}=\frac{1}{\hat{T}}, \quad \hat{q}=\frac{q}{p \sqrt{\frac{k}{m} T_{W_{0}}}}, \quad K n=\frac{{\sqrt{\frac{k}{m} T_{W_{0}}}}^{3}}{\alpha L p} .
\end{aligned}
$$

There remains the following simple system of ordinary non-linear differential equations

$$
\frac{5}{2} \frac{d \hat{T}}{d \hat{x}}+\frac{1}{6} \frac{d \hat{\Delta}}{d \hat{x}}=-\frac{2}{3 K_{n}} \frac{\hat{q}}{\hat{T}}, \frac{d \hat{T}}{d \hat{x}}=-\frac{1}{42 K_{n}} \frac{\hat{\Delta}}{\hat{q} \hat{T}} \text { and } \hat{q}=\text { const } .
$$

For a given Knudsen number $K n$ (i.e. given pressure), the solution of (36) requires three boundary conditions. In an experiment, however, we can control only two quantities, viz. the temperatures $T_{W_{0}}$ and $T_{W_{L}}$ of the walls [1].

The jump conditions (35) relate the wall temperatures and the values of the moments in front of the walls, denoted by $T_{0}, \Delta_{0}$ and $T_{L}, \Delta_{L}$, respectively. In dimensionless form we find the relations

$$
\hat{T}_{W_{0}}-\hat{T}_{0}=\frac{\frac{2-\theta}{2 \theta} \sqrt{\frac{\pi}{2}} \sqrt{\hat{T}_{0}} \hat{q}+\frac{1}{30} \hat{\Delta}_{0}}{1-\frac{1}{120} \frac{\hat{\Delta}_{0}}{\hat{T}_{0}}}, \quad \hat{T}_{W_{L}}-\hat{T}_{L}=\frac{-\frac{2-\theta}{2 \theta} \sqrt{\frac{\pi}{2}} \sqrt{\hat{T}_{L}} \hat{q}+\frac{1}{30} \hat{\Delta}_{L}}{1-\frac{1}{120} \frac{\hat{\Delta}_{L}}{\hat{T}_{L}}} .
$$

With these boundary conditions, the system (36) could be solved numerically, if the heat flux $\hat{q}$ were known. $\hat{q}$ enters these equations as a parameter. Indeed, we need one additional boundary value for the heat flux. In an experiment, however, it is impossible to prescribe the heat flux and the two temperatures of the walls independently.

Thus, additional assumptions are needed for the determination of the heat flux. For heat transfer without temperature jumps Struchtrup \& Weiss [1] have determined the heat flux from the postulate that the maximum of the local entropy production becomes minimal in stationary processes. We shall use the same method and a similar one, which assumes that the global entropy production becomes minimal in stationary processes [18] [19]. The results of both methods will be compared to calculations with so-called kinetic schemes [2].

For given values $\hat{T}_{W_{0}}, \hat{T}_{W_{L}}$ and $\hat{q}$ the equations (36) and (37) were solved numerically. Note, that not all choices of the heat flux lead to realistic results. We must require

$$
\hat{T}_{W_{0}} \leq \hat{T}_{0} \leq \hat{T}(x) \leq \hat{T}_{L} \leq \hat{T}_{W_{L}} .
$$

Indeed, for given values $\hat{T}_{W_{0}}, \hat{T}_{W_{L}}, K n$ and $\theta$, these inequalities hold only within a small interval $\left(\hat{q}_{\min }, \hat{q}_{\max }\right)$ of values for $\hat{q}$. For $\hat{T}_{W_{0}}=1, \hat{T}_{W_{L}}=2$, and $\theta=1$, the table below gives the appropriate values of $\hat{q}_{\min }, \hat{q}_{\max }$.

\begin{tabular}{lll}
\hline$K n$ & $\hat{q}_{\max }$ & $\hat{q}_{\min }$ \\
\hline 0.1 & -0.26436 & -0.30705 \\
0.01 & -0.050519 & -0.051682 \\
\hline
\end{tabular}

We have defined temperature as a measure for the kinetic energy of the gas, see (2), but the notion of temperature in non-equilibrium is not clear, see [20] for discussion and the introduction of an appropriate thermodynamic temperature. 
In order to find the relation to extended thermodynamics with 13 moments, we eliminate $\hat{\Delta}$ from (36),

$$
\hat{q}=-\frac{15}{4} K n \hat{T} \frac{d \hat{T}}{d \hat{x}}+\frac{21}{2} K n^{2} \hat{q} \hat{T} \frac{d}{d \hat{x}}\left[\hat{T} \frac{d \hat{T}}{d \hat{x}}\right]
$$

For small Knudsen numbers, the second term on the r.h.s. may be ignored. Thus (38) reduces to Fourier's law (27). In the case of small Knudsen numbers, the 13 field theory is sufficient for a proper description of stationary heat transfer.

\subsection{Kinetic schemes solutions}

Stationary heat transfer with 14 moments was also considered in [2] by means of the so-called kinetic schemes [16]. The results of [2] are not fully satisfactory since the heat flux is not a constant but jumps at the walls so that the energy conservation law is violated. It is therefore imperative to compare the results of [2] with the numerical solution of (36), (37), which has a constant heat flux at the walls by construction.
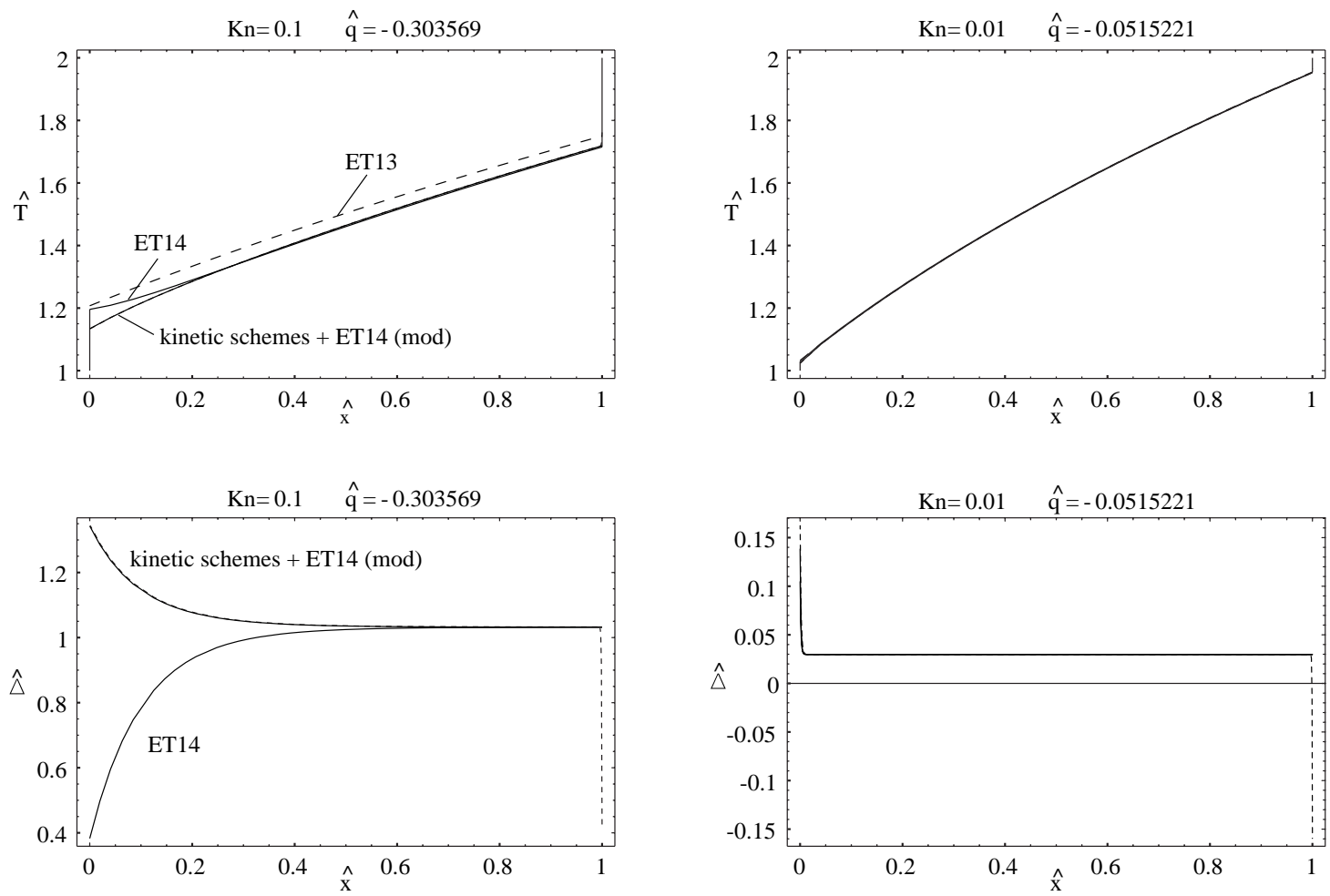

Fig. 2. Comparison of kinetic scheme results with solution of (36), (37) with $\hat{T}_{W_{0}}=1, \hat{T}_{W_{L}}=2$ (ET14) and with modified wall temperatures (ET14 (mod)). The curve ET13 shows the corresponding solution of (32). Left column: Kn=0.1. Right column: Kn=0.01, all curves lie on top of each other.

As examples we consider the case of full accommodation, $\theta=1$, with wall temperatures $\hat{T}_{W_{0}}=1, \hat{T}_{W_{L}}=2$ and Knudsen numbers $K n=0.1, K n=0.01$.

The kinetic scheme calculations with $1.000(K n=0.1)$ and $10.000(K n=0.01)$ grid points provide a heat flux in the interior $\hat{q}(K n=0.1)=-0.303569$ and $\hat{q}(K n=0.01)=-0.0515221$, respectively. In order to compare the kinetic scheme results with the solution of the equations (36), (37), we have solved the equations for the same values of $\hat{q}$. Note, that in this case no additional boundary conditions are needed, since the value for $\hat{q}$ follows from the kinetic scheme solution. If we cannot rely on these, however, the value of $\hat{q}$ must be determined by an additional entropy principle, see below. The results of the kinetic scheme and the equations (36), (37) for the same values of $\hat{q}$ are shown in Fig. 2. 
For $K n=0.1$ (left column) there are marked differences in $T$ and $\Delta$ in the interval $x \in\{0,0.3\}$. These are due to the above mentioned problems of the kinetic scheme with conservation of energy at the walls. In order to find a measure for the error, we have computed - by (37) - new wall temperatures from the kinetic scheme data and found $\hat{T}_{W_{0}}=0.97453, \hat{T}_{W_{L}}=2.00428$. With these modified boundary data, the solution of (36) gives exactly the kinetic scheme solution. Thus, at this Knudsen number, the kinetic scheme introduces an error of approximately $3 \%$ in the boundary conditions.

For the smaller Knudsen number $K n=0.01$ the difference of the kinetic scheme and the present solution is very small. Here, the modified temperatures are $T_{W_{0}}=0.99600$ and $T_{W_{L}}=1.99842$ and the corresponding curves agree very well (right column of Fig. 2). Indeed, in the figures all curves lie on top of each other. The most remarkable difference is found in the values of $\Delta$ at $x=0$ with $\hat{\Delta}_{\text {0num }}=0.104705$ for the numerical solution and $\hat{\Delta}_{0 \mathrm{kS}}=0.16231$ from the kinetic scheme. Moreover, only the latter has the steep descent of $\hat{\Delta}$ at $x=1$. However, these differences have no influence on the results for the temperature which - due to the small Knudsen number $K n=0.01$ - agrees very well with the 13 field solution (32).

We conclude that the kinetic schemes are not correct in the interesting case of large Knudsen numbers, but introduce an error at the boundaries, see [2] for a detailed discussion. Thus, the kinetic schemes results cannot serve as a proper benchmark for the present problem. However, they may approximately give the proper results, and we shall therefore still compare our results with those of the kinetic scheme in the next sections.

\subsection{Entropy productions}

Let us remember that the previous solution of (36), (37) was based on the value of $q$ from the kinetic scheme calculations. Now we consider the two afore-mentioned principles for the entropy production which will allow us to find $q$.

In order to apply the entropy production principles, we need the local entropy production density (per unit volume) which is given by [5]

$$
\Sigma=-k \int \ln \frac{f}{y} \mathscr{S}(f) d \mathbf{c}
$$

where $1 / y$ is the volume of a cell of phase space. In the case of stationary heat transfer with 14 moments we obtain in dimensionless form

$$
\hat{\Sigma}=\frac{4}{15 K_{n}} \frac{\hat{q}^{2}}{\hat{T}^{3}}+\frac{1}{180 K_{n}} \frac{\hat{\Delta}^{2}}{\hat{T}^{4}}
$$

Note, that $\hat{\Sigma}$ is an approximation of the entropy production, where only quadratic contributions in the nonequilibrium quantities $q$ and $\Delta$ are taken into account.

While there is no production of energy and momentum at the walls - heat flux and tangential stress are constant - there is a production of entropy at the walls [15]. For the following we assume that this contribution (a production density per unit area) does not influence the behavior of the gas between the walls. Thus, we shall consider only the entropy production (40).

\subsection{Global principle for the entropy production}

The application of the global principle for the entropy production works as follows:

1. Choose the parameters which are given or controllable, i.e. the wall temperatures $\hat{T}_{W_{0}}, \hat{T}_{W_{L}}$, the Knudsen number $K n$ and the accommodation coefficient $\theta$.

2. Choose a value for the heat flux $\hat{q}$.

3. Solve the boundary value problem (36) with (37). This yields the fields $\hat{T}(\hat{x} ; \hat{q}), \hat{\Delta}(\hat{x} ; \hat{q})$. 
4. Calculate the entropy production density $\hat{\Sigma}(\hat{x} ; \hat{q})$ and compute the global entropy production (per unit area)

$$
\hat{\Sigma}_{\text {tot }}(\hat{q})=\int_{0}^{1} \hat{\Sigma}(\hat{x} ; \hat{q}) d \hat{x}
$$

5. Return to 3.) and change $\hat{q}$. Proceed until $\min \left[\hat{\Sigma}_{\text {tot }}(\hat{q})\right]$ is found.

According to the global principle, the heat flux corresponding to $\min \left[\hat{\Sigma}_{\mathrm{tot}}(\hat{q})\right]$ is assumed to be measured in an experiment. Results will be presented below.

\subsection{Minimax principle for the entropy production}

The application of the minimax principle for the entropy production works as follows:

1. Choose the parameters which are given or controllable, i.e. the wall temperatures $\hat{T}_{W_{0}}, \hat{T}_{W_{L}}$, the Knudsen number $K n$ and the accommodation coefficient $\theta$.

2. Choose a value for the heat flux $\hat{q}$.

3. Solve the boundary value problem (36) with (37). This yields the fields $\hat{T}(\hat{x} ; \hat{q}), \hat{\Delta}(\hat{x} ; \hat{q})$.

4. Calculate the entropy production density $\hat{\Sigma}(\hat{x} ; \hat{q})$ and compute its maximum

$$
\hat{\Sigma}_{\max }(\hat{q})=\max [\hat{\Sigma}(\hat{x} ; \hat{q})] .
$$

5. Return to 3.) and change $\hat{q}$. Proceed until $\min \left[\hat{\Sigma}_{\max }(\hat{q})\right]$ is found.

According to the minimax principle, the heat flux corresponding to $\min \left[\hat{\Sigma}_{\max }(\hat{q})\right]$ is assumed to be the one that is found in an experiment.

\subsection{Results}

Again, we consider the case of full accommodation, $\theta=1$ with wall temperatures $\hat{T}_{W_{0}}=1, \hat{T}_{W_{L}}=2$ and Knudsen numbers $K n=0.1, K n=0.01$.

Figure 3 shows the results for $K n=0.1$. In the left column we compare the results from the minimax principle $\left(\hat{q}_{\operatorname{minimax}}=-0.310263\right)$ with the results from the kinetic scheme calculations of Sect. 6.3, while the right column shows the results from the global principle $\left(\hat{q}_{\text {global }}=-0.29207\right)$ in comparison with the kinetic scheme. The pictures in the first row show the temperature, including the dashed temperature curve of the 13 field case (32). The latter - due to the large Knudsen number - differs from the other three temperature curves which stem from the 14-moment case. Again, we find differences between the results which are small in case of the temperature but clearly visible in case of $\Delta$, where the kinetic scheme and the global principle predict a decreasing curve, while the minimax principle predicts an increasing curve. Thus, the qualitative agreement between the result of the global principle and the kinetic scheme is better then the agreement between the results from the minimax principle in comparison to the kinetic scheme.

We have also used the corrected wall temperatures of Sect. 6.3 and found a good agreement between the kinetic scheme solution $\left(\hat{q}_{\mathrm{kS}}=-0.303569\right)$ and the result of the global principle $\left(\hat{q}_{\mathrm{global}}=-0.29207\right)$ with decreasing $\Delta$, while with the minimax principle $\left(\hat{q}_{\operatorname{minimax}}=-0.310236\right) \Delta$ is increasing.

The dimensionless fields $\hat{q}$ and $\hat{\Delta}$ are of order unity for this large Knudsen number so that the approximation (40) for the entropy production is not proper. The results from both principles can only give an indication and therefore we do not discuss the finer details as the existence of two local maxima for the entropy production.

Moreover, we like to emphasize that the kinetic scheme results - due to the violation of conservation laws at the walls - should not be overestimated. In particular they cannot be employed to decide which of the two entropy principles should be preferred. 
Figure 4 shows temperature $\hat{T}$ and entropy production $\hat{\Sigma}$ for the case $K n=0.01$ where the quadratic entropy production (40) is appropriate. Here, the temperature curves of the minimax principle, kinetic scheme and 13-moment match very well. The corresponding heat fluxes $-\hat{q}_{\operatorname{minimax}}=-0.0516442, q_{k s}=-0.0515236$, $\hat{q}_{13}=-0.0516414$ - differ by less then $0.3 \%$. The result from the global principle, however, differs by about $5 \%: \hat{q}_{\text {global }}=-0.0490105$. Differences can be found in the values of $\Delta$ at $x=0$ with $\hat{\Delta}_{0 \operatorname{minimax}}=-0.00555326$, $\hat{\Delta}_{\text {oglobal }}=2.20706, \hat{\Delta}_{0 \mathrm{kS}}=0.17626$
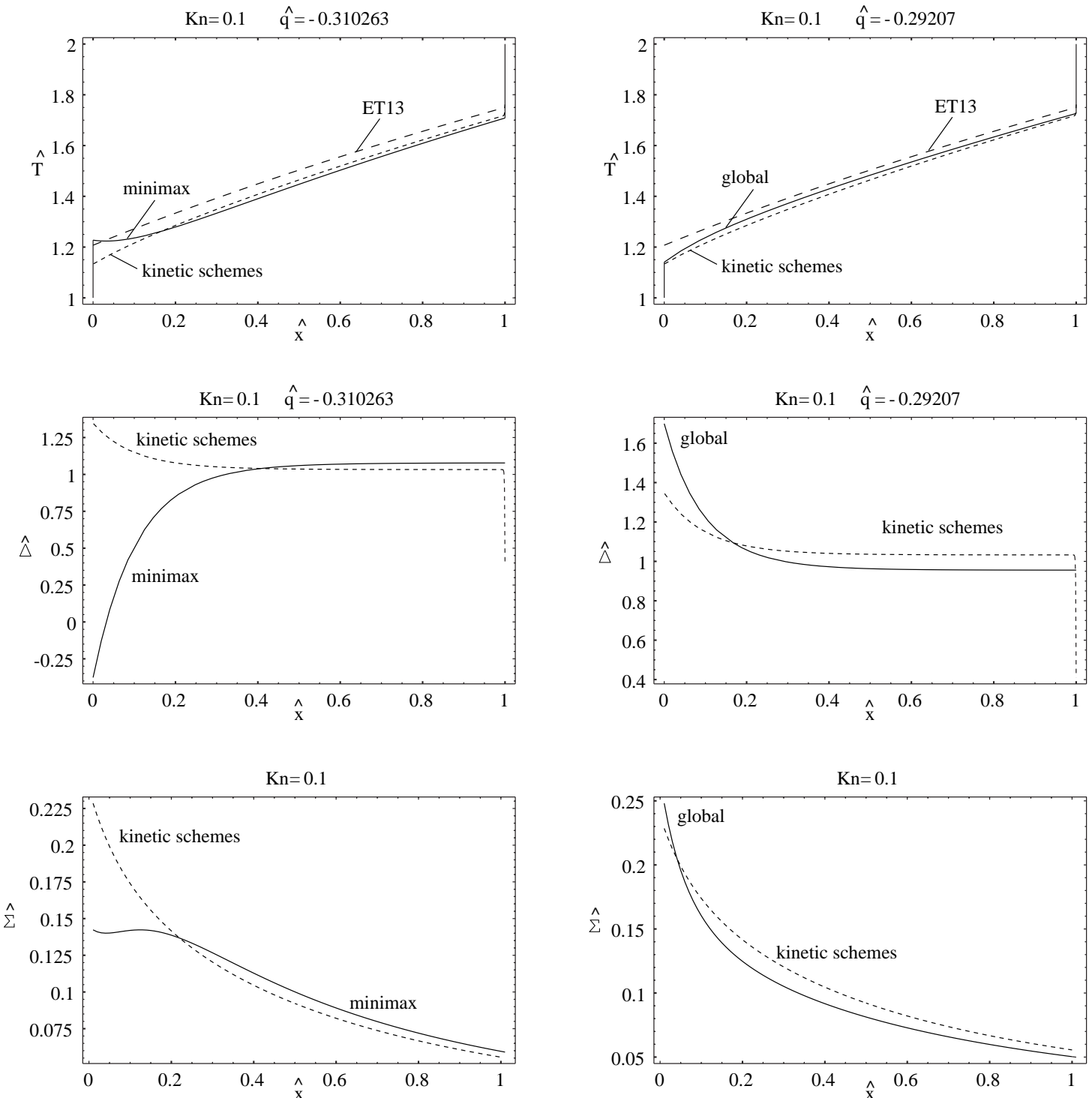

Fig. 3. Temperature $T$, fourth moment $\Delta$ and entropy production $\Sigma$ for $K n=0.1$. Left column: solution according to the minimax principle in comparison to solution of kinetic scheme. Right column: solution according to the global minimum principle in comparison to solution of kinetic scheme. Also the temperature curve for the analytic solution with 13 moments (32) is shown.

The global principle favours a solution with an additional temperature jump at the left which is accompanied by a sharp increase in the entropy production. The large entropy production at the very left allows for a smaller entropy production at the other space points, where the gas is closer to equilibrium. The minimax principle does not permit this behavior but leads to a solution where the entropy productions at neighboring space points are more balanced. These findings are the same as in [1] for the case without jumps. 

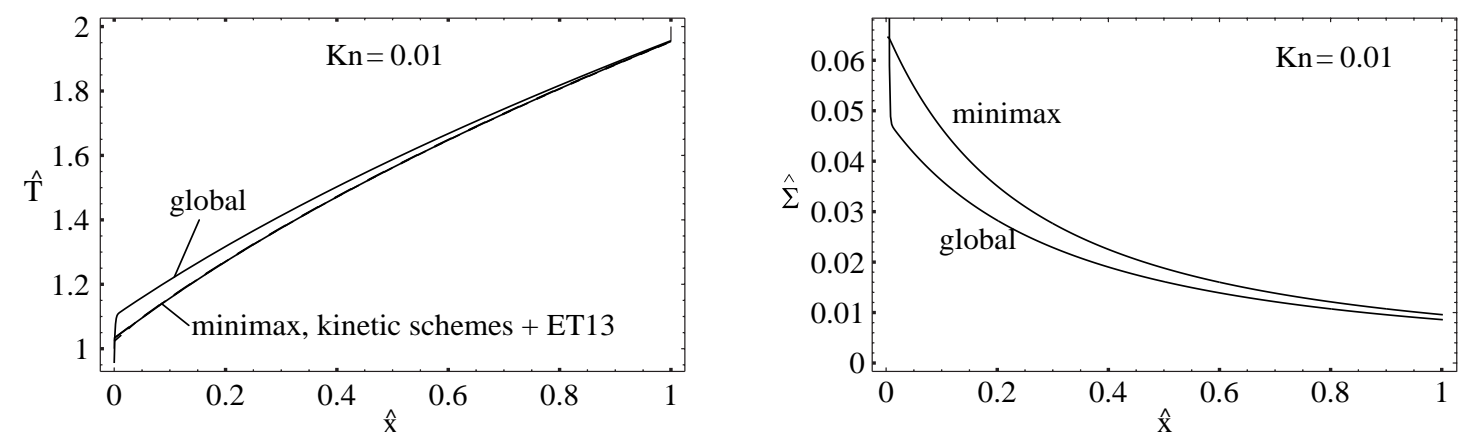

Fig. 4. Temperature $T$ and entropy production $\Sigma$ for $K n=0.01$. Solutions according to the minimax principle, the global minimum principle, kinetic schemes, and analytic solution for 13 moments (32).

For smaller Knudsen numbers the global principle leads to increasingly bad results with a sharp increase of the temperature at the left while the minimax principle agrees very well with the 13 field result, which is appropriate for small Knudsen numbers, see (38).

In summary we must state that the minimax principle gives reasonable results for all Knudsen numbers while the global principle fails for small $\mathrm{Kn}$.

\section{Conclusions}

In this paper we have set up equations which relate temperature jump and slip at a wall to the values of the moments inside the gas. These were used for the solution of the stationary heat transfer problem for the 13 and the 14 moment case. While for the 13 moment case no additional boundary conditions are necessary, the 14 moment case requires an additional boundary condition for the heat flux which was determined from the minimax principle for the local entropy production and the minimum principle for the global entropy production.

Our results indicate that the global principle should not be used: its results are wrong at low Knudsen numbers; most probably the reasonable results for large Knudsen numbers are coincidental.

The minimax principle gives good results for all Knudsen numbers. In particular for small Kn it agrees with the analytical solution for the 13 field case. For large Knudsen numbers, it does not give the same predictions as the kinetic scheme of [2]. This may be due to the inaccuracies of the latter but there are also reasons to have some doubt about the minimax results. Indeed, for large $K n$, the non-equilibrium quantities $\hat{q}$ and $\hat{\Delta}$ are not small but of order unity in dimensionless form. Thus, the entropy production (40), which is quadratic in $q$ and $\Delta$, does not give the proper entropy production - clearly this causes an error. Here, one should rely on a numerical computation of the entropy production integral (39).

We state again that 14 moments will not be sufficient for large $K n$ so that the corresponding results have a restricted physical meaning. It is not clear, which and how many moments one will need for the proper description of the gas in case of large Knudsen numbers. According to our experience with the moment method and extended thermodynamics the moment number should be much bigger than 14 . In particular, one has to expect the formation of boundary layers at the walls, which are only weakly pronounced in the case of 14 moments, where they stem from the non-linearities in the moment equations. Thus, we may expect proper results only in case of a larger moment number. Once these are obtained we may compare with results from Monte Carlo methods or molecular dynamics. Calculations of the heat transfer experiment with higher moment numbers are in preparation. We are confident that these will give better insight into the nature and usefulness of the minimax principle for the entropy production as well as in the properties of the kinetic schemes. 


\section{A Half-space moments of the Maxwellian}

This appendix is devoted to the calculation of the half-space-moments of the Maxwellian, which are defined by

$$
h_{i_{1} \cdots i_{n}}^{r}=m \int_{n_{i} C_{i} \geq 0} C^{2 r} C_{i_{1}} \cdots C_{i_{n}} f_{M} d \mathbf{C},
$$

where $f_{M}$ is the Maxwellian phase density

$$
f_{M}=\frac{\varrho}{m} \sqrt{\frac{m}{2 \pi k T}}^{3} e^{-\frac{m}{2 k T} C^{2}} .
$$

With the dimensionless velocity $x=\sqrt{\frac{m}{2 k T}} C$ and the direction vector

$$
\nu_{i}=C_{i} / C=\{\cos \varphi \sin \vartheta, \sin \varphi \sin \vartheta, \cos \vartheta\}_{i},
$$

where $n_{i} \nu_{i}=\cos \vartheta$, we find

$$
\begin{aligned}
& h_{i_{1} \cdots i_{n}}^{r}=\frac{\varrho}{\sqrt{\pi}^{3}}\left(\frac{2 k T}{m}\right)^{r+\frac{n}{2}} \int_{x=0}^{\infty} e^{-x^{2}} x^{2 r+2+n} d x \int_{\varphi=0}^{2 \pi} \int_{\vartheta=0}^{\pi / 2} \nu_{i_{1}} \cdots \nu_{i_{n}} \sin \vartheta d \vartheta d \varphi \\
& h_{i_{1} \cdots i_{n}}^{r}=\varrho\left(\frac{2 k T}{m}\right)^{r+\frac{n}{2}} \frac{\Gamma\left(r+\frac{3+n}{2}\right)}{\sqrt{\pi}} I_{i_{1} \cdots i_{n}} .
\end{aligned}
$$

Here, we have introduced the abbreviation

$$
I_{i_{1} \cdots i_{n}}=\frac{1}{2 \pi} \int_{\varphi=0}^{2 \pi} \int_{\vartheta=0}^{\pi / 2} \nu_{i_{1}} \cdots \nu_{i_{n}} \sin \vartheta d \vartheta d \varphi .
$$

We proceed with the calculation of the integrals for various values of $n$.

For $n=0$ we obtain easily

$$
I=\frac{1}{2 \pi} \int_{\varphi=0}^{2 \pi} \int_{\vartheta=0}^{\pi / 2} \sin \vartheta d \vartheta d \varphi=1
$$

so that we have for the scalar half-space moments

$$
h^{r}=\varrho\left(\frac{2 k T}{m}\right)^{r} \frac{\Gamma\left(r+\frac{3}{2}\right)}{\sqrt{\pi}} .
$$

The direction of the normal $n_{i}$ is the only distinguished vector in the calculation of the half-space moments. Thus we must have for the vector integral

$$
I_{i}=\frac{1}{2 \pi} \int_{\varphi=0}^{2 \pi} \int_{\vartheta=0}^{\pi / 2} \nu_{i} \sin \vartheta d \vartheta d \varphi=\gamma n_{i} .
$$

Scalar multiplication with $n_{i}$ gives

$$
\gamma=\frac{1}{2 \pi} \int_{\varphi=0}^{2 \pi} \int_{\vartheta=0}^{\pi / 2} \cos \vartheta \sin \vartheta d \vartheta d \varphi=\frac{1}{2}
$$

and we find the vectorial moments as

$$
h_{i}^{r}=\varrho\left(\frac{2 k T}{m}\right)^{r+\frac{1}{2}} \frac{\Gamma(r+2)}{2 \sqrt{\pi}} n_{i} .
$$

The tensorial integral has the form 


$$
I_{i j}=\frac{1}{2 \pi} \int_{\varphi=0}^{2 \pi} \int_{\vartheta=0}^{\pi / 2} \nu_{i} \nu_{j} \sin \vartheta d \vartheta d \varphi=\gamma n_{i} n_{j}+\beta \delta_{i j}
$$

where the coefficients follow by scalar multiplication with $n_{i} n_{j}$ and $\delta_{i j}$ as $\gamma=0$ and $\beta=1 / 3$ so that

$$
h_{i j}^{r}=\varrho\left(\frac{2 k T}{m}\right)^{r+1} \frac{\Gamma\left(r+\frac{5}{2}\right)}{3 \sqrt{\pi}} \delta_{i j} .
$$

For the integral with three indices we find the representation

$$
I_{i j k}=\frac{1}{2 \pi} \int_{\varphi=0}^{2 \pi} \int_{\vartheta=0}^{\pi / 2} \nu_{i} \nu_{j} \nu_{k} \sin \vartheta d \vartheta d \varphi=\gamma n_{i} n_{j} n_{k}+\beta\left(n_{i} \delta_{j k}+n_{j} \delta_{i k}+n_{k} \delta_{i j}\right),
$$

where the coefficients follow by multiplication with $n_{i} n_{j} n_{k}$ and $n_{i} \delta_{j k}$, respectively. We obtain

$$
\gamma+3 \beta=\frac{1}{4} \text { and } \gamma+5 \beta=\frac{1}{2} \text { so that } \beta=-\gamma=\frac{1}{8}
$$

and

$$
h_{i j k}^{r}=\varrho\left(\frac{2 k T}{m}\right)^{r+\frac{3}{2}} \frac{\Gamma(r+3)}{8 \sqrt{\pi}}\left(n_{i} \delta_{j k}+n_{j} \delta_{i k}+n_{k} \delta_{i j}-n_{i} n_{j} n_{k}\right) .
$$

The fourth order integral has the representation

$$
\begin{aligned}
I_{i j k l}= & \alpha\left(\delta_{i j} \delta_{k l}+\delta_{i k} \delta_{j l}+\delta_{i l} \delta_{j k}\right)+\gamma n_{i} n_{j} n_{k} n_{l}+ \\
& +\beta\left(n_{i} n_{j} \delta_{k l}+n_{i} n_{k} \delta_{j l}+n_{i} n_{l} \delta_{j k}+n_{j} n_{k} \delta_{i l}+n_{j} n_{l} \delta_{i k}+n_{k} n_{l} \delta_{i j}\right) .
\end{aligned}
$$

Multiplication with $n_{i} n_{j} n_{k} n_{l}, n_{i} n_{j} \delta_{k l}$ and $\delta_{i j} \delta_{k l}$ yields $\beta=\gamma=0$ and $\alpha=1 / 15$ so that

$$
h_{i j k l}^{r}=\varrho\left(\frac{2 k T}{m}\right)^{r+2} \frac{\Gamma\left(r+\frac{7}{2}\right)}{15 \sqrt{\pi}}\left(\delta_{i j} \delta_{k l}+\delta_{i k} \delta_{j l}+\delta_{i l} \delta_{j k}\right) .
$$

\section{B Half-space moments for 13 and 14 moments}

In extended thermodynamics of 14 fields one finds the phase density

$$
\begin{aligned}
f_{14}= & f_{M}\left(1+\frac{m^{2}}{8 \varrho k^{2} T^{2}} \Delta-\frac{m^{3}}{12 \varrho k^{3} T^{3}} \Delta C^{2}+\frac{m^{4}}{120 \varrho k^{4} T^{4}} \Delta C^{4}-\right. \\
& \left.+\frac{m^{2}}{2 \varrho k^{2} T^{2}} p_{\langle j k\rangle} C_{j} C_{k}-\frac{m^{2}}{\varrho k^{2} T^{2}} q_{k} C_{k}\left(1-\frac{1}{5} \frac{m}{k T} C^{2}\right)\right)
\end{aligned}
$$

which reduces to Grad's phase density for $\Delta=0$. The half-space moments of this function are defined by

$$
H_{i_{1} \cdots i_{n}}^{r}=m \int_{n_{i} C_{i} \geq 0} C^{2 r} C_{i_{1}} \cdots C_{i_{n}} f_{14} d \mathbf{C}
$$

and may easily be expressed in terms of the half-space moments of the Maxwellian which we have calculated in Appendix A. We obtain

$$
\begin{aligned}
H_{i_{1} \cdots i_{n}}^{r}= & h_{i_{1} \cdots i_{n}}^{r}+\frac{m^{2}}{8 \varrho k^{2} T^{2}} \Delta h_{i_{1} \cdots i_{n}}^{r}-\frac{m^{3}}{12 \varrho k^{3} T^{3}} \Delta h_{i_{1} \cdots i_{n}}^{r+1}+\frac{m^{4}}{120 \varrho k^{4} T^{4}} \Delta h_{i_{1} \cdots i_{n}}^{r+2}- \\
& +\frac{m^{2}}{2 \varrho k^{2} T^{2}} p_{\langle j k\rangle} h_{i_{1} \cdots i_{n} j k}^{r}-\frac{m^{2}}{\varrho k^{2} T^{2}} q_{k} h_{i_{1} \cdots i_{n} k}^{r}+\frac{1}{5} \frac{m^{3}}{\varrho k^{3} T^{3}} q_{k} h_{i_{1} \cdots i_{n} k}^{r+1} .
\end{aligned}
$$

The half-space moments of Grad's phase density follow for $\Delta=0$. 


\section{References}

1. Struchtrup, H., Weiss, W. (1998) Maximum of the local entropy production becomes minimal in stationary processes. Phys. Rev. Lett. 80, 5048-5051

2. Struchtrup, H. Kinetic schemes and boundary conditions for moment equations. To appear in J. Appl. Math. Phys. (2000) (ZAMP)

3. Grad, H. (1949) On the Kinetic Theory of Rarefied Gases. Comm. Pure Appl. Math., Vol.2, Wiley, New York

4. Dreyer, W. (1987) Maximization of the Entropy in Non-equilibrium. J. Phys. A: Math. Gen. 20, 6505

5. Müller, I., Ruggeri, T. (1993) Rational Extended Thermodynamics. Springer, New York (Springer Tracts in Natural Philosophy Vol. 37) $\left(2^{\text {nd }}\right.$ ed. 1998)

6. Jou, D., Casas-Vásquez, J., Lebon, G. (1996) Extended Irreversible Thermodynamics. Springer, Berlin

7. Levermore, C.D. (1996) Moment Closure Hierarchies for Kinetic Theories. J. Stat. Phys. 83, 1021-1065

8. Weiss, W. (1990) Zur Hierarchie der Erweiterten Thermodynamik. Dissertation, TU Berlin

9. Weiss, W. (1995) Continuous shock structure in extended thermodynamics. Phys. Rev. E 52, 5760

10. Struchtrup, H. (1997) An Extended Moment Method in Radiative Transfer: The Matrices of Mean Absorption and Scattering Coefficients. Ann. Phys. 257, 111-135

11. Struchtrup, H. (1998) On the number of moments in radiative transfer problems. Ann. Phys. 266, 1-26

12. Dreyer, W., Struchtrup, H. (1993) Heat Pulse Experiments Revisited. Cont. Mech. Thermodyn. 5, 3-50

13. De Groot, S.R., Mazur, P. (1969) Non-equilibrium Thermodynamics. North-Holland, Amsterdam

14. Chapman, S., Cowling, T.G. (1970) The mathematical Theory of Non-Uniform Gases. Cambridge University Press

15. Cercignani, C. (1975) Theory and Application of the Boltzmann Equation. Scottish Academic Press

16. LeTallec, P., Perlat, J.P. (1997) Numerical Analysis of Levermore’s Moment System. INRIA preprint N 3124

17. Reitebuch, D., Weiss, W. (1999) Application of High Moment Theory to the Plane Couette Flow. Cont. Mech. Thermodyn. 4, 217-225

18. Prigogine, I. (1967) Introduction to Thermodynamics of Irreversible Processes. Interscience, New York

19. Glansdorff, P., Progogine, I. (1964) On a general evolution criterion in macroscopic physics. Physica 30, 351-374

20. Barbera, E., Müller, I., Sugiyama, M. (1999) On the Temperature of a Rarefied Gas in Non-Equilibrium. Meccanica 34, 103-113 (1999) 